\title{
Nanosensors enable early detection of acute $T$ cell-mediated rejection of transplants
}

T cell-mediated rejection (TCMR) remains an important cause of graft failure in kidney transplant recipients. Its early detection is essential for effective management; however, diagnosis of TCMR currently requires biopsy. Now, a collaborative effort has led to the development of nanosensors that enable the early, noninvasive detection of TCMR in skin allografts. "At the time I met transplant clinicians Dave Mathews and Andrew Adams my laboratory was thinking about developing immune sensors to monitor T cell activity, but in conversations it became clear that an immediate and impactful application would be to focus on the early detection of graft rejection," explains Gabriel Kwong, a biomedical engineer. "It has been a nice example of a collaborative effort between the two groups with Dr Kwong's expertise in nanotechnology and $T$ cell activity and our experience in $\mathrm{T}$ cell immunology and transplantation," adds Adams.

Biopsy is the gold-standard approach for the diagnosis of allograft rejection; however, it is invasive and only detects damage once it has occurred. "Most of the time the decision to proceed with biopsy is only triggered when

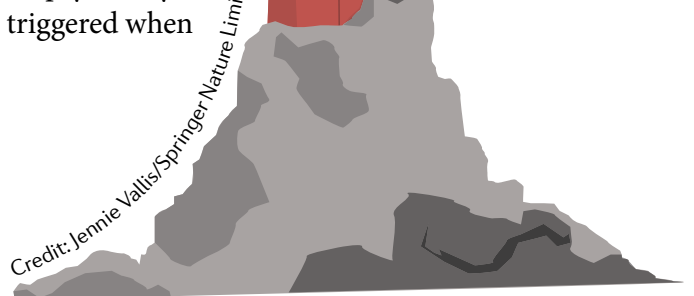

dysfunction is detected on routine laboratory findings, at which point

Our technology

can detect the beginnings of the rejection

response before organ damage occurs there is already some level of damage to the transplanted organ," explains Adams. "Our technology can detect the beginnings of the rejection response before organ damage occurs."

To develop a nanosensor that detects early TCMR, the researchers conjugated iron oxide nanoparticles with a peptide substrate specific for the serine protease granzyme B. Previous work had shown that recipient $\mathrm{T}$ cells produce granzyme $\mathrm{B}$ at the onset of TCMR, and that the presence of granzyme B-positive $\mathrm{T}$ cells predicts progression to severe TCMR. Using a mouse model of skin graft rejection, Kwong, Adams and colleagues show that following systemic administration, the nanosensors preferentially accumulate in allograft tissue owing to localized vasodilation at the site of inflammation. Once the nanosensors have reached the site of anti-graft $T$ cell activity, they are cleaved by $\mathrm{T}$ cell-derived granzyme $\mathrm{B}$, which releases fluorescent reporters that are subsequently filtered into the urine, where they are detected. Importantly, the nanosensors could detect an increase in granzyme B activity in transplanted mice several days before TCMR was evident histologically. "Our approach was to design immune sensors that are administered by a simple intravenous push and directly query transplants for signs of anti-graft immune responses," says Kwong. "Filtration of the detection signals into the transplant recipient's urine allows us to noninvasively analyse urine samples to determine graft health. In the future, we envision that this approach might enable routine monitoring of patients without the risk and discomfort of a biopsy."

To assess the sensitivity of the nanosensors in detecting $T$ cell activity under conditions of immunosuppression, the researchers performed urinalysis of transplanted mice treated with the calcineurin inhibitor tacrolimus. Although tacrolimus treatment improved graft outcomes, treatment was subtherapeutic and did not prevent eventual graft loss in major histocompatibility complex-mismatched recipients. Administration of the nanonsensor demonstrated elevated granzyme $B$ activity, which was predictive of eventual graft failure in these mice.

The researchers say that they are continuing their work to develop new nanosensors for the detection of other forms of allograft rejection. "We envision building a panel of immune sensors that may eventually enable not just early detection, but also discrimination between the different types of rejection to guide treatment decisions" says Kwong. "The most exciting aspect of this technology is the ability to potentially distinguish the cause of $\mathrm{T}$ cell activation or other immune responses," adds Adams. "Allograft dysfunction can also be caused by infections such as BK virus, ischaemic injury or antibody-mediated injury. The ability to have various substrates for each of these scenarios could identify the cause of injury and the correct course of treatment."

Susan J. Allison

ORIGINAL ARTICLE Mac, O. D. et al.

Non-invasive early detection of acute transplant rejection via nanosensors of granzyme B activity. Nat. Biomed. Eng. https://doi.org/10.1038/

541551-019-0358-7 (2019) 